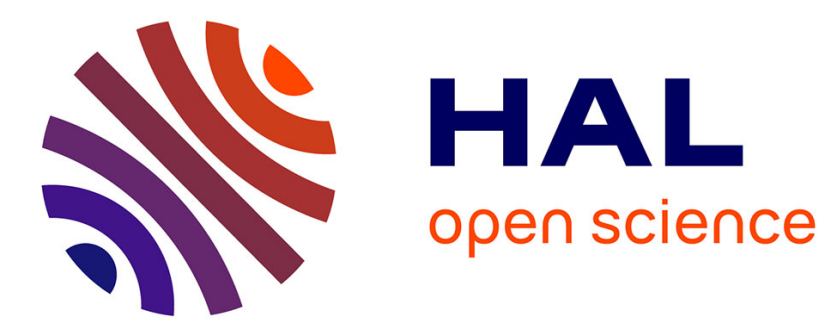

\title{
Coordinating partially-informed agents over state-dependent networks
}

Benjamin Larrousse, Samson Lasaulce, Michèle Wigger

\section{To cite this version:}

Benjamin Larrousse, Samson Lasaulce, Michèle Wigger. Coordinating partially-informed agents over state-dependent networks. 2015 IEEE Information Theory Workshop (ITW), Apr 2015, Jérusalem, Israel. 10.1109/ITW.2015.7133152 . hal-01272502

\section{HAL Id: hal-01272502 https://hal.science/hal-01272502}

Submitted on 15 Feb 2016

HAL is a multi-disciplinary open access archive for the deposit and dissemination of scientific research documents, whether they are published or not. The documents may come from teaching and research institutions in France or abroad, or from public or private research centers.
L'archive ouverte pluridisciplinaire $\mathbf{H A L}$, est destinée au dépôt et à la diffusion de documents scientifiques de niveau recherche, publiés ou non, émanant des établissements d'enseignement et de recherche français ou étrangers, des laboratoires publics ou privés. 


\section{Coordinating Partially-Informed Agents over State-Dependent Networks}

\author{
Benjamin Larrousse \\ Centrale-Supelec \\ 91192 Gif-sur-Yvette, France \\ benjamin.larrousse@1ss.supelec.fr
}

\author{
Samson Lasaulce \\ CNRS and Centrale-Supelec \\ 91192 Gif-sur-Yvette, France \\ lasaulce@1ss.supelec.fr
}

\author{
Michèle Wigger \\ Telecom ParisTech \\ 75013 Paris, France \\ michele.wigger@telecom-paristech.fr
}

\begin{abstract}
We consider a multi-agent scenario with $K \geq 2$ agents that have partial information about some random nature state, and that take actions in a repeated manner. Each agent also has imperfect observations of the other agents' past actions and the nature state realization. Our goal is to characterize the set of asymptotically implementable distributions on the agents' actions and the nature state. We solve this problem for general $K$ when all agents have only causal nature state information (NSI) and for $K=2$ when: one agent has causal NSI and the other agent has non-causal NSI; or in some special cases when both agents have non-causal NSI.
\end{abstract}

\section{INTRODUCTION}

This paper solves a few special instances of the following general problem. Consider $K \geq 2$ agents, where Agent $k \in\{1, \ldots, K\}$ produces time- $t$ action $^{1} x_{k, t} \in \mathcal{X}_{k}$ for $t \in\{1, \ldots, T\}, T \geq 1$. Each agent has access to some observations associated with the chosen actions and the realization of a random process $\left\{S_{0, t}\right\}_{t=1}^{T}=\left\{S_{0,1}, \ldots, S_{0, T}\right\} \in \mathcal{S}_{0}^{T}$, which models some process of interest. We call $\left\{S_{0, t}\right\}$ the nature states. The agents' actions and the nature states also affect the agents' individual payoff functions $\left\{\omega_{k}\right\}_{k=1}^{K}$, where $\omega_{k}: \mathcal{S}_{0} \times \mathcal{X}_{1} \times \ldots \times \mathcal{X}_{K} \rightarrow \mathbb{R}$. The main problem under investigation is to characterize, for a given observation structure, the set of feasible average payoffs

$$
W_{k}^{(T)}=\frac{1}{T} \sum_{t=1}^{T} \omega_{k}\left(s_{0, t}, x_{1, t}, \ldots, x_{K, t}\right), k \in\{1, \ldots, K\},
$$

in the limit $T \rightarrow \infty$. Let $P_{S_{0, t} X_{1, t} \ldots X_{K, t}}$ denote the joint distribution on the $(K+1)$-tuple $\left(S_{0}, X_{1}, \ldots, X_{K}\right)$ at time $t$. By definition, the set of feasible average payoffs is fully characterized by the set of averaged distributions $\frac{1}{T} \sum_{t=1}^{T} P_{S_{0, t} X_{1, t} \ldots X_{K, t}}\left(s_{0}, x_{1}, \ldots, x_{K}\right)$ that can be induced by the agents' strategies. To reuse the terminology of [1], [2], in the limit $T \rightarrow \infty$, this set will be referred to as the set of implementable distributions (see Definition 1). Characterizing the set of implementable distributions for this general problem is a challenging problem, which motivates the definition of intermediate problems such as those reported in this paper.

The first special instance of the general problem described above is due to Gossner et al. [1], who addressed the two-agent case when: the nature states $\left\{S_{0, t}\right\}_{t=1}^{T}$ are i.i.d. (independent

\footnotetext{
${ }^{1}$ Throughout the paper, we assume that all alphabets such as $\mathcal{X}_{k}$ are finite.
}

and identically distributed); at any time $t \in\{1, \ldots, T\}$, Agent 1 knows the past, present, and future realizations of the nature state $\left(s_{0,1}, \ldots, s_{0, T}\right)$; Agent 2 knows the past realizations of the nature state $\left(s_{0,1}, \ldots, s_{0, t-1}\right)$; and both Agents 1 and 2 have perfect observation of the past actions $\left(x_{1,1}, x_{2,1}, \ldots, x_{1, t-1}, x_{2, t-1}\right)$. The problem is solved in [1]; Cuff and Zhao presented an alternative proof [3] of the results in [1] based on more traditional information-theoretic tools and under the coordination via actions framework. In [2], the authors address the more general case where at time $t \in\{2, \ldots, T\}$ Agent 2 has access to the past realizations of nature state $\left(s_{0,1}, s_{0,2}, \ldots, s_{0, t-1}\right)$ and to observations $\left(y_{2,1}, y_{2,2}, \ldots, y_{2, t-1}\right)$ that are modeled as the outputs of a discrete memoryless channel (DMC) $\Gamma\left(y_{2, t} \mid x_{1, t}\right)$; this setup has independently been addressed in [4]. The work of [2] was further developed in $[5,6]$, where at time $t$ Agent 2 has access to the past outputs $\left(y_{2,1}, \ldots, y_{2, t-1}\right)$ of a more general statedependent DMC $\Gamma\left(y_{2, t} \mid s_{0, t}, x_{1, t}, x_{2, t}\right)$, but not necessarily to the past realizations $\left(s_{0,1}, \ldots, s_{0, t-1}\right)$ of the nature state.

In contrast to the above works, in the present paper we consider more symmetric scenarios where each agent $k \in\{1,2\}$ has nature state information (NSI) about $\left\{S_{0, t}\right\}$ and observes past outputs $\left(y_{k, 1}, \ldots, y_{k, t-1}\right)$ from a state-dependent discrete memoryless network $\Gamma\left(y_{1, t}, y_{2, t} \mid s_{0, t}, x_{1, t}, x_{2, t}\right)$. Moreover, the NSI at the two agents can be only partial. We also make a step toward the multi-agent scenario with $K>2$ by discussing the complexity of the problem and providing first results.

\section{General Problem formulation}

We explain our assumptions in more detail. The nature states $\left\{S_{0, t}\right\}_{t=1}^{T}$ are i.i.d. according to the law $\rho_{0}$. Each Agent $k$ 's NSI, $k \in\{1, \ldots, K\}$, is modeled by the sequence $\left\{S_{k, t}\right\}_{t=1}^{T} \in \mathcal{S}_{k}^{T}$, where the $(K+1)$-tuples $\left\{\left(S_{0, t}, S_{1, t}, \ldots, S_{K, t}\right)\right\}_{t=1}^{T}$ are i.i.d. according to the joint distribution $\rho_{0}\left(s_{0}\right) \times \bar{\top}\left(s_{1}, \ldots, s_{K} \mid s_{0}\right)$, with $s_{k} \in \mathcal{S}_{k}$. The observations $\left\{Y_{k, t}\right\}$ at Agent $k$ are best modeled through a state-dependent network that at each time $t$ takes as inputs the agents' actions $X_{1, t}, \ldots, X_{K, t}$ and the nature state $S_{0, t}$, and produces as outputs the observations $Y_{1, t}, \ldots, Y_{K, t}$. We assume that this network is memoryless and is described by a stationary channel law $\Gamma\left(y_{1}, \ldots, y_{K} \mid s_{0}, x_{1}, \ldots, x_{K}\right)$, i.e., that ${ }^{2}$

\footnotetext{
${ }^{2}$ Throughout this manuscript, we adopt the notations $A^{n}$ and $a^{n}$ for the $n$-tuples $\left(A_{1}, \ldots, A_{n}\right)$ and $\left(a_{1}, \ldots, a_{n}\right)$ and $A_{m}^{n}$ for $\left(A_{m}, \ldots, A_{n}\right)$.
} 


$$
\operatorname{Pr}\left[Y_{1, t}=y_{1, t}, Y_{2, t}=y_{2, t} \mid S_{0}^{t}=s_{0}^{t}, X_{1}^{t}=x_{1}^{t}, X_{2}^{t}=x_{2}^{t}, Y_{1}^{t-1}=y_{1}^{t-1}, Y_{2}^{t-1}=y_{2}^{t-1}\right]=\Gamma\left(y_{1, t}, y_{2, t} \mid s_{0, t}, x_{1, t}, x_{2, t}\right)
$$

(2) on top of the next page holds. We distinguish between two scenarios. In the first scenario, Agent $k$ has causal NSI, i.e., it produces its time- $t$ action $X_{k, t}$ as

$$
X_{k, t}=\sigma_{k, t}^{(\mathrm{c})}\left(S_{k}^{t}, Y_{k}^{t-1}\right)
$$

for some $\sigma_{k, t}^{(\mathrm{c})}: \mathcal{S}_{k}^{t} \times \mathcal{Y}_{k}^{t-1} \rightarrow \mathcal{X}_{k}$. The sequence $\left\{\sigma_{k, t}^{(\mathrm{c})}\right\}_{1 \leq t \leq T}$ is also referred to as the strategy of Agent $k$ under causal NSI. In the second scenario, Agent $k$ has non-causal NSI, i.e., it produces its time- $t$ action $X_{k, t}$ as

$$
X_{k, t}=\sigma_{k, t}^{(\mathrm{nc})}\left(S_{k}^{T}, Y_{k}^{t-1}\right)
$$

for some $\sigma_{k, t}^{(\mathrm{nc})}: \mathcal{S}_{k}^{T} \times \mathcal{Y}_{k}^{t-1} \rightarrow \mathcal{X}_{k}$.

Remark 1: The scenario where an agent $k$ has strictly-causal $N S I$ is contained in the scenario where the agent has no NSI at all, because strictly-causal NSI can always be modeled with an enhanced output $\widetilde{Y}_{k} \triangleq\left(Y_{k}, S_{k}\right)$.

The main issue at stake is to find the average joint distributions that can asymptotically be induced by some strategy profilei.e., by some vector of strategies at agents $1, \ldots, K$. This is precisely the notion of implementability defined next.

Definition 1: Fix $\left(\mathrm{c}_{1}, \ldots, \mathrm{c}_{K}\right) \in\{\mathrm{c}, \mathrm{nc}\}^{K}$. A probability distribution $\bar{Q}\left(s_{0}, x_{1}, \ldots, x_{K}\right)$ is implementable if for every $\epsilon>0$ and sufficiently large $T \geq 1$ there exists a strategy profile $\left(\sigma_{1, t}^{\mathrm{c}_{1}}, \ldots, \sigma_{K, t}^{\mathrm{c}_{\mathrm{K}}}\right)_{1 \leq t \leq T}$ which induces at each time $t$ a joint distribution $P_{S_{0, t} X_{1, t} \cdots X_{K, t}}$ on the $(K+1)$-uple $\left(S_{0, t}, X_{1, t}, \ldots, X_{K, t}\right)$ such that for all $\left(s_{0}, x_{1}, x_{2}, \ldots, x_{K}\right) \in$ $\mathcal{S}_{0} \times \mathcal{X}_{1} \times \mathcal{X}_{2} \times \cdots \times \mathcal{X}_{K}$ :

$$
\begin{aligned}
& \mid \frac{1}{T} \sum_{t=1}^{T} P_{S_{0, t} X_{1, t} \cdots X_{K, t}}(\left.s_{0}, x_{1}, x_{2}, \ldots, x_{K}\right) \\
&-\bar{Q}\left(s_{0}, x_{1}, x_{2}, \ldots, x_{K}\right) \mid \leq \epsilon .
\end{aligned}
$$

Definition 1 implies that a distribution $\bar{Q}\left(s_{0}, x_{1}, \ldots, x_{K}\right)$ can only be implementable if $\sum_{x_{1}, \ldots, x_{K}} \bar{Q}\left(s_{0}, x_{1}, \ldots, x_{K}\right)=$ $\rho_{0}\left(s_{0}\right)$. The main purpose of this paper is to characterize the set of implementable distributions, which we will denote $\mathcal{Q}$.

\section{Results for the Two-Agent Case}

In this section we focus on $K=2$ agents. We first characterize the set of implementable distributions $\mathcal{Q}$ when both agents have causal NSI. As our result illustrates, in this case $\mathcal{Q}$ is not increased by communicating over the network.

Theorem 1: Let $\mathrm{c}_{1}=\mathrm{c}_{2}=\mathrm{c}$. The set of implementable distributions $\mathcal{Q}$ consists of the marginal distributions

$$
\begin{aligned}
\bar{Q}\left(s_{0}, x_{1}, x_{2}\right)=\sum_{u, s_{1}, s_{2}} & {\left[\rho_{0}\left(s_{0}\right)\right\urcorner\left(s_{1}, s_{2} \mid s_{0}\right) P_{U}(u) } \\
\times & \left.P_{X_{1} \mid U S_{1}}\left(x_{1} \mid u, s_{1}\right) P_{X_{2} \mid U S_{2}}\left(x_{2} \mid u, s_{2}\right)\right]
\end{aligned}
$$

where $P_{U}$ and $\left\{P_{X_{k} \mid U S_{k}}\right\}_{k=1}^{2}$ are arbitrary (conditional) distributions over a finite set $\mathcal{U}$ and over $\mathcal{X}_{1}$ and $\mathcal{X}_{2}$.

Proof: Omitted.

Communication over the network can enlarge the set of implementable distributions $\mathcal{Q}$ when at least one of the two agents has non-causal NSI. At first we assume that only Agent 1 has non-causal NSI while Agent 2 has causal NSI.

Theorem 2: Let $\mathrm{c}_{1}=\mathrm{nc}$ and $\mathrm{c}_{2}=\mathrm{c}$. In this case the set of implementable distributions $\mathcal{Q}$ consists of the marginals $\bar{Q}\left(s_{0}, x_{1}, x_{2}\right)$ of all joint distributions $Q\left(s_{0}, s_{1}, s_{2}, u, v, x_{1}, x_{2}, y_{1}, y_{2}\right)$ that factorize as

$$
\begin{aligned}
& Q\left(s_{0}, s_{1}, s_{2}, u, v, x_{1}, x_{2}, y_{1}, y_{2}\right) \\
& =\rho_{0}\left(s_{0}\right) \backslash\left(s_{1}, s_{2} \mid s_{0}\right) P_{U V X_{1} \mid S_{1}}\left(u, v, x_{1} \mid s_{1}\right) \\
& P_{X_{2} \mid U S_{2}}\left(x_{2} \mid u, s_{2}\right) \Gamma\left(y_{1}, y_{2} \mid s_{0}, x_{1}, x_{2}\right)
\end{aligned}
$$

and that satisfy the mutual information constraint ${ }^{3}$

$$
I_{Q}\left(S_{1} ; U\right) \leq I_{Q}\left(V ; Y_{2} \mid U\right)-I_{Q}\left(V ; S_{1} \mid U\right) .
$$

Proof: Omitted.

In the special case where Agent 2 has no NSI at all i.e., $S_{2}=$ const. and Agent 1 has perfect NSI $S_{1}=S_{0}$, Theorem 2 recovers a result in [5] [6]; in this case the auxiliary random variable $U$ can be replaced by Agent 2's action $X_{2}$.

It can be seen that the set of implementable distributions $\mathcal{Q}$ in Theorem 2 depends only on Agent 2's observation $Y_{2}$ but not $Y_{1}$. This shows that a communication from Agent 2-who has only causal NSI - to Agent 1 does not enlarge $\mathcal{Q}$.

In Proposition 1 we extend the strategy profiles leading to Theorem 2 to a symmetric scenario where both agents have non-causal NSI. A key feature of the new strategies is that the agents use a Wyner-Ziv source-code [7] instead of a standard source-code. For this reason the left-hand side of (6b) in Proposition 1 shows the Wyner-Ziv source-coding rate $I\left(S_{1} ; U_{1} \mid S_{2}\right)$ whereas the left-hand side of $(5 \mathrm{~b})$ in Theorem 2 shows the standard source-coding rate $I\left(S_{1} ; U\right)$.

Proposition 1: Let $\mathrm{c}_{1}=\mathrm{c}_{2}=\mathrm{NC}$. The set of implementable distributions $\mathcal{Q}$ contains the marginals $\bar{Q}\left(s_{0}, x_{1}, x_{2}\right)$ of all joint distributions $Q\left(s_{0}, s_{1}, s_{2}, u_{1}, u_{2}, v_{1}, v_{2}, x_{1}, x_{2}, y_{1}, y_{2}\right)$ that factorize as

$$
\begin{aligned}
Q\left(s_{0},\right. & \left.s_{1}, s_{2}, u_{1}, u_{2}, v_{2}, v_{1}, x_{1}, x_{2}, y_{1}, y_{2}\right) \\
= & \left.\rho_{0}\left(s_{0}\right)\right\urcorner\left(s_{1}, s_{2} \mid s_{0}\right) Q_{U_{1} \mid S_{1}}\left(u_{1} \mid s_{1}\right) Q_{U_{2} \mid S_{2}}\left(u_{2} \mid s_{2}\right) \\
& \times Q_{X_{1} V_{1} \mid U_{1} S_{1}}\left(x_{1}, v_{1} \mid u_{1}, s_{1}\right) Q_{X_{2} V_{2} \mid U_{2} S_{2}}\left(x_{2}, v_{2} \mid u_{2}, s_{2}\right) \\
& \times \Gamma\left(y_{1}, y_{2} \mid s_{0}, x_{1}, x_{2}\right)
\end{aligned}
$$

and that satisfy the mutual information constraints

$$
\begin{aligned}
& \quad I_{Q}\left(S_{1} ; U_{1} \mid S_{2}\right) \\
& \quad \leq I_{Q}\left(V_{1} ; Y_{2}, S_{2}, V_{2} \mid U_{1}, U_{2}\right)-I_{Q}\left(V_{1} ; S_{1} \mid U_{1}, U_{2}\right)
\end{aligned}
$$

\footnotetext{
${ }^{3}$ The notation $I_{Q}(A ; B)$ indicates that the mutual information should be
} computed with respect to the probability distribution $Q$. 


$$
\begin{aligned}
& I_{Q}\left(S_{2} ; U_{2} \mid S_{1}\right) \\
& \quad \leq I_{Q}\left(V_{2} ; Y_{1}, S_{1}, V_{1} \mid U_{2}, U_{2}\right)-I_{Q}\left(V_{2} ; S_{2} \mid U_{1}, U_{2}\right) .
\end{aligned}
$$

Proof: See Section V.

We have a matching converse (Theorem 3 ) to this Proposition 1 for two special cases where Agent 2 cannot convey any interesting information to Agent 1. Either because there is no way to communicate, see Condition (7), or because Agent 1 is already aware of Agent 2's NSI, see Condition (8).

The special case meeting Condition (7) is of particular interest to power control problems in interference channels [2]. Indeed, it can be checked that (7) is verified when $s_{0}$ denotes the global channel state information, $x_{k}$ the transmit power of transmitter $k$, and $y_{k}$ the ratio between the transmitter $k$ 's signal power and receiver $k$ 's interference-plus-noise power.

Theorem 3: Let $\mathrm{c}_{1}=\mathrm{c}_{2}=\mathrm{nc}$. Assume further that one of the following two conditions holds:

- the network transition law (2) is of the form

$$
\Gamma\left(y_{1}, y_{2} \mid s_{0}, x_{1}, x_{2}\right)=\widetilde{\Gamma}\left(y_{1}, y_{2} \mid s_{0}, x_{1}\right)
$$

for some conditional probability distribution $\widetilde{\Gamma}$; or

- Agent 1 can compute Agent 2's NSI because

$$
S_{2, t}=f\left(S_{1, t}\right), \quad t \in\{1, \ldots, T\},
$$

for some function $f: \mathcal{S}_{1} \rightarrow \mathcal{S}_{2}$.

In this case, the subset of $\mathcal{Q}$ described in Proposition 1 is exactly the set of implementable distributions $\mathcal{Q}$.

Proof: See Section VI.

For general scenarios the set of implementable distributions presented in Proposition 1 should be enlarged if the strategies in Section V are modified so as to employ Kaspi's two-way source coding [8] instead of non-interactive Wyner-Ziv coding.

Remark 2: In many applications, an agent might have both causal and non-causal NSI. For example, when the state $\left\{S_{0, t}\right\}$ models the weather at some location, on the one hand the agent can obtain rough non-causal NSI from annual statistics, and on the other hand it obtains much more reliable causal NSI by observing the weather at its own location or through weather forecasts. Our model and results are easily generalized in this sense. In particular, for the scenario with mixed causal and non-causal CIS at both agents, the set of implementable distributions $\mathcal{Q}$ can be derived when Condition (7) or (8) holds.

\section{IV. $K \geq 2$ AGEnTs}

\section{A. Causal NSI at all agents}

Theorem 1 readily generalizes to arbitrary $K \geq 2$. Indeed, it can be proved that the corresponding distributions write as $\bar{Q}\left(s_{0}, x_{1}, \ldots, x_{K}\right)=$ $\sum_{u, s_{1}, \ldots, s_{k}}\left[\left(\prod_{k=1}^{K} \mathrm{P}_{X_{k} \mid U S_{k}}\left(x_{k} \mid u, s_{k}\right)\right) \times P_{U}(u) \times\right.$ $\neg\left(s_{1}, \ldots, s_{k} \mid s_{0}\right) \times \rho_{0}\left(s_{0}\right)$. Thus, as for two agents, communication does not enlarge the set of implementable distributions $\mathcal{Q}$ when the agents only have causal NSI.

\section{B. Non-causal NSI at all agents}

The general problem is very hard, and includes in particular the general joint source-channel coding problem as a special case. This follows from the following proposition:

Proposition 2: Consider a network with transition law $\Gamma$ that neither depends on the state $S_{0}$ nor on the actions of Agents $j+1, \ldots, K$. That means, for some conditional law $\widetilde{\Gamma}$ :

$\Gamma\left(y_{1}, \ldots, y_{K} \mid s_{0}, x_{1}, \ldots, x_{K}\right)=\widetilde{\Gamma}\left(y_{1}, \ldots, y_{K} \mid x_{1}, \ldots, x_{j}\right)$.(9)

Assume non-causal NSI at all Agents $1, \ldots, K\left(c_{1}=\cdots=\right.$ $\mathrm{c}_{K}=\mathrm{nc}$ ) and that we are interested only in coordinating the actions of Agents $j+1, \ldots, K$ with state $s_{0}$.

In this case, the set of implementable distributions is unchanged if Agents $j+1, \ldots, K$ can wait to produce their actions until they have observed all their channel outputs, i.e.,

$$
X_{k, t}=\tilde{\sigma}_{k, t}\left(S_{k}^{T}, Y_{k}^{T}\right), \quad k=j+1, \ldots, K .
$$

Proof: Omitted.

Notice that the joint source-channel coding problem is open for almost all networks, including the "simple" 3-node multi-access, broadcast, and relay channels. Characterizing the set of implementable distributions $\mathcal{Q}$ even for these 3node networks thus seems out of reach. Based on the strategy profiles presented in Section $\mathrm{V}$, one can however readily identify subsets of $\mathcal{Q}$.

To see this, we briefly recall the strategy profiles presented in Section V. They operate over subblocks of length $n$. Agent $k$ constructs its subblock- $b$ actions $X_{k,(b-1) n+1}, \ldots, X_{k, b n}$ by first Wyner-Ziv source-coding its subblock- $(b+1)$ state sequence $S_{k, b n+1}, \ldots, S_{k,(b+1) n}$ and then Gel'fand-Pinsker channel-coding [9] the resulting compression index. ${ }^{4}$ At the end of block $b$, Agent $k$ decodes the other agent's Gelfand-Pinsker code and reconstructs the compressed version of the other agent's block- $(b+1)$ state sequence. Thus, each agent knows the compressed versions of both block- $(b+1)$ state sequences $\left(S_{1, b n+1}, \ldots, S_{1,(b+1) n}\right)$ and $\left(S_{2, b n+1}, \ldots, S_{2,(b+1) n}\right)$ already before block $b+1$ starts. It can thus use these compressed sequences as additional sideinformation in the block- $(b+1)$ Gel'fand-Pinsker codes.

These strategies can be employed over any general network. They can be improved, for example, by replacing the pointto-point Gel'fand-Pinsker codes through a network Gel'fandPinsker code $[11,12]$ that is tailored to the structure of the network. Or the Wyner-Ziv compression can be replaced by a network source-code [13]-[15] or coordination-code [16].

For some choices of the network Gel'fand-Pinsker codes or the network source-codes, the overall coding idea has to be slightly modified, for example by introducing an additional block of look-ahead in the forward encoding. This is in particular the case for the following cascade scenario with 3 agents, where the channel law $\Gamma$ decomposes as

$$
\Gamma\left(y_{2}, y_{3} \mid s_{0}, x_{1}, x_{2}\right)=\Gamma_{1}\left(y_{2} \mid s_{0}, x_{1}\right) \cdot \Gamma_{2}\left(y_{3} \mid s_{0}, x_{2}\right),
$$

\footnotetext{
${ }^{4}$ This idea of forward-encoding the state informations has previously been used, e.g, in $[1,3,10]$.
} 
and where Agent 1 has non-causal NSI $S_{1, t}=S_{0, t}$ and Agents 2 and 3 have no NSI at all. We let Agent 1 use the optimal cascade source-code [15] to compress each $b$-th subblock of its state sequence, $S_{0,(b-1) n+1}, \ldots, S_{0, b n}$, and to send the resulting two compression indices in block $b-2$ to Agent 2. Agent 2 then forwards one the two compression indices to Agent 3 in the next block $b-1$. The sketched strategy profile leads to the following:

Proposition 3 (3-agent cascade network): The set of implementable distributions $\mathcal{Q}$ for the described scenario contains the marginals $\bar{Q}\left(s_{0}, x_{1}, x_{2}, x_{3}\right)$ of all joint distributions $Q\left(s_{0}, v_{2}, v_{3}, x_{1}, x_{2}, x_{3}, y_{1}, y_{2}\right)$ that factorize as

$$
\begin{aligned}
Q\left(s_{0}, v_{2}, v_{3}, x_{1}, x_{2}, x_{3}, y_{2}, y_{3}\right) \\
=\bar{Q}\left(s_{0}, x_{1}, x_{2}, x_{3}\right) Q_{V_{1} V_{2} \mid S_{0} X_{1} X_{2} X_{3}}\left(v_{1}, v_{2} \mid s_{0}, x_{1}, x_{2}, x_{3}\right) \\
\quad \times \Gamma_{1}\left(y_{2} \mid s_{0}, x_{1}\right) \Gamma_{2}\left(y_{3} \mid s_{0}, x_{2}\right)
\end{aligned}
$$

and that satisfy the following two conditions

$$
\begin{gathered}
I_{Q}\left(S_{0} ; X_{2}, X_{3}\right) \leq I_{Q}\left(V_{1} ; Y_{2} \mid X_{2}, X_{3}\right)-I_{Q}\left(V_{1} ; S_{0}, X_{2} \mid X_{3}\right) \\
I_{Q}\left(S_{0} ; X_{3}\right) \leq I_{Q}\left(V_{2} ; Y_{3} \mid X_{3}\right)-I_{Q}\left(V_{2} ; S_{0} \mid X_{3}\right) .
\end{gathered}
$$

Proof: Omitted.

The above subset of $\mathcal{Q}$ indeed coincides with $\mathcal{Q}$ in the special case when $\Gamma_{2}$ does not depend on $s_{0}$. In general the subset inclusion is however strict.

\section{Strategy Profile achieving Proposition 1}

Choose distribution $Q\left(s_{0}, s_{1}, s_{2}, u_{1}, u_{2}, v_{1}, v_{2}, x_{1}, x_{2}, y_{1}, y_{2}\right)$ satisfying the three conditions (6) in the theorem.

Fix small $\epsilon_{3}>\epsilon_{2}>\epsilon_{1}>0$, and pick positive rates $R_{1}, R_{2}, \tilde{R}_{1}, \tilde{R}_{2}, R_{1}^{\prime}, R_{2}^{\prime}$ so that

$$
\begin{aligned}
R_{1}+ & R_{1}^{\prime}>I\left(S_{1} ; U_{1}\right) \\
& R_{1}^{\prime}<I\left(U_{1} ; S_{2}\right) \\
& \tilde{R}_{1}>I\left(V_{1} ; S_{1}, U_{1}, U_{2}\right) \\
R_{1}+\tilde{R}_{1} & <I\left(V_{1} ; Y_{2}, S_{2}, U_{1}, U_{2}, V_{2}\right) .
\end{aligned}
$$

and

$$
\begin{aligned}
R_{2}+ & R_{2}^{\prime}>I\left(S_{2} ; U_{2}\right) \\
R_{2}^{\prime} & <I\left(U_{2} ; S_{1}\right) \\
\tilde{R}_{2} & >I\left(V_{2} ; S_{2}, U_{2}, U_{1}\right) \\
R_{2}+\tilde{R}_{2} & <I\left(V_{2} ; Y_{1}, S_{1}, U_{1}, U_{2}, V_{1}\right) .
\end{aligned}
$$

By conditions (6b) and (6c) such rates exist.

Codebooks generation: Split the blocklength $T$ into $B$ blocks each of length $n \triangleq\lfloor T / B\rfloor$. For each block $b \in\{1, \ldots, B\}$ and each Agent $k \in\{1,2\}$ randomly generate a codebook $\mathcal{C}_{U_{k}}^{(b)}$ containing the $n$-length codewords $\left\{u_{k}^{(b)}\left(i_{k}, \nu_{k}\right)\right\}$ for $i_{k} \in\left\{1, \ldots,\left\lfloor 2^{n R_{k}}\right\rfloor\right\}$ and $\nu_{k} \in$ $\left\{1, \ldots,\left\lfloor 2^{n R_{k}^{\prime}}\right\rfloor\right\}$ by drawing all entries of all codewords i.i.d. according to the marginal distribution $Q_{U_{k}}{ }^{5}$ Independent thereof, randomly generate for each block $b \in\{1, \ldots, B\}$

\footnotetext{
${ }^{5}$ In this section all marginals are meant with respect to the joint distribution $Q\left(s_{0}, s_{1}, s_{2}, u_{1}, u_{2}, v_{2}, v_{1}, x_{1}, x_{2}, y_{1}, y_{2}\right)$ in (6a)
}

and each Agent $k \in\{1,2\}$ a codebook $\mathcal{C}_{V_{k}}^{(b)}$ containing the $n$ length codewords $\left\{v_{k}^{(b)}\left(j_{k}, \ell_{k}\right)\right\}$ for $j_{k} \in\left\{1, \ldots,\left\lfloor 2^{n R_{k}}\right\rfloor\right\}$ and $\ell_{k} \in\left\{1, \ldots,\left\lfloor 2^{n \tilde{R}_{k}}\right\rfloor\right\}$ by drawing all entries of all codewords i.i.d. according to the marginal distribution $Q_{V_{k}}$.

For each block $b \in\{1, \ldots, B\}$, let $s_{1}^{(b)}$ and $s_{2}^{(b)}$ denote the state informations at Agents 1 and 2 corresponding to this block $b$.

Strategies at Agent $k \in\{1,2\}$ : Let $i_{k}^{(1)}=j_{k}^{(B)}=1$. Before transmission starts, for each block $b \in\{2, \ldots, B\}$, Agent $k$ looks for a pair of indices $i_{k}^{(b)} \in\left\{1, \ldots,\left\lfloor 2^{n R_{k}}\right\rfloor\right\}$ and $\nu_{k}^{(b)} \in$ $\left\{1, \ldots,\left\lfloor 2^{n R_{k}^{\prime}}\right\rfloor\right\}$ so that

$$
\left(s_{k}^{(b)}, u_{k}^{(b)}\left(i_{k}^{(b)}, \nu_{k}^{(b)}\right)\right) \in \mathcal{T}_{\epsilon_{1}}^{n}\left(Q_{S_{k} U_{k}}\right) .
$$

If there is more than one such pair of indices, Agent 1 chooses one of them at random, otherwise it declares an error. It then sets $j_{k}^{(b-1)}=i_{k}^{(b)}$.

Now, at the start of each block $b \in\{1, \ldots, B\}$, just before it has to produce its time $(b-1) n+1$ input $X_{k,(b-1) n+1}$, Agent $k$ looks for an index $\ell_{k}^{(b)} \in\left\{1, \ldots,\left\lfloor 2^{n \tilde{R}_{k}}\right\rfloor\right\}$ such that

$$
\begin{aligned}
\left(s_{k}^{(b)}, u_{k}^{(b)}\left(i_{k}^{(b)}, \nu_{k}^{(b)}\right), u_{\bar{k}}^{(b)}\left(\hat{i}_{\bar{k}}^{(b)}, \hat{\nu}_{\bar{k}}^{(b)}\right), v_{k}^{(b)}\left(j_{k}^{(b)}, \ell_{k}^{(b)}\right)\right) \\
\\
\in \mathcal{T}_{\epsilon_{2}}^{n}\left(Q_{S_{k} U_{k} U_{\bar{k}} V_{k}}\right),
\end{aligned}
$$

where $\bar{k}$ is the index in $\{1,2\}$ not equal to $k$ and-as we will see shortly- $\hat{i}_{\bar{k}}^{(b)}$ and $\hat{\nu}_{\bar{k}}^{(b)}$ are indices that Agent $k$ produced at the end of the previous block $(b-1)$. If there is at least one such index $\ell_{k}^{(b)}$, Agent $k$ picks one of these candidates at random, otherwise it declares an error.

In this block $b$, Agent $k$ produces its actions as follows. For each $\tau \in\{1, \ldots, n\}$, Agent $k$ creates $X_{k,(b-1) n+\tau}$ by applying the conditional law $Q_{X_{k} \mid S_{k} U_{k} U_{\bar{k}} V_{k}}$ to the $\tau$-th components of the codewords $u_{k}^{(b)}\left(i_{k}^{(b)}, \nu_{k}^{(b)}\right), u_{\bar{k}}^{(b)}\left(\hat{i}_{\bar{k}}^{(b)}, \hat{\nu}_{\bar{k}}^{(b)}\right), v_{k}^{(b)}\left(j_{k}^{(b)}, \ell_{k}^{(b)}\right)$ and to its state information $s_{k,(b-1) n+\tau}$.

At the end of this block $b$, after observing the last channel output $y_{k, b n}$, Agent $k$ also looks for a pair of indices $\left.\left(\hat{j}_{\bar{k}}^{(b)}, \hat{\ell}_{\bar{k}}^{(b)}\right)\right) \in\left\{1, \ldots,\left\lfloor 2^{n R_{k}}\right\rfloor\right\} \times\left\{1, \ldots,\left\lfloor 2^{n \tilde{R}_{k}}\right\rfloor\right\}$ such that

$$
\begin{aligned}
\left(s_{k}^{(b)}, u_{k}^{(b)}\left(i_{k}^{(b)}, \nu_{k}^{(b)}\right),\right. & u_{\bar{k}}^{(b)}\left(\hat{i}_{\bar{k}}^{(b)}, \hat{\nu}_{\bar{k}}^{(b)}\right), \\
v_{k}^{(b)}\left(j_{k}^{(b)}, \ell_{k}^{(b)}\right), & \left.v_{\bar{k}}^{(b)}\left(\hat{j}_{\bar{k}}^{(b)}, \hat{\ell}_{\bar{k}}^{(b)}\right), y_{k}^{(b)}\right) \\
& \in \mathcal{T}_{\epsilon_{3}}^{n}\left(Q_{S_{k} U_{k} U_{\bar{k}} V_{k} V_{\bar{k}} Y_{k}}\right) .
\end{aligned}
$$

If there is at least one such pair of indices, it picks one of them at random. Otherwise it declares an error.

Agent $k$, further sets $\hat{i}_{\bar{k}}^{(b+1)}=\hat{j}_{\bar{k}}^{(b)}$ and looks for an index $\hat{\nu}_{\bar{k}}^{(b+1)}\left\{1, \ldots,\left\lfloor 2^{n R_{k}^{\prime}}\right\rfloor\right\}$ such that

$$
\left(s_{k}^{(b+1)}, u_{\bar{k}}^{(b+1)}\left(\hat{i}_{\bar{k}}^{(b+1)}, \hat{\nu}_{\bar{k}}^{(b+1)}\right)\right) \in \mathcal{T}_{\epsilon_{2}}^{n}\left(Q_{U_{\bar{k}} S_{k}}\right) .
$$

Analysis: Omitted.

\section{Proof of Theorem 3}

Let $\bar{Q}\left(s_{0}, x_{1}, x_{2}\right)$ be an implementable distribution. Fix $\epsilon>$ 0 . By definition, there exists a sufficiently large blocklength $T$ and strategies $\left\{\sigma_{1, t}^{(\mathrm{nc})}\right\}_{t=1}^{T}$ and $\left\{\sigma_{2, t}^{(\mathrm{nc})}\right\}_{t=1}^{T}$ such that for each 
$t \in\{1, \ldots, T\}$, the tuple $\left(S_{0, t}, X_{1, t}, X_{2, t}\right)$ induced by these strategies has a joint law $P_{S_{0, t} X_{1, t} X_{2, t}}$ that satisfies

$$
\left|\frac{1}{T} \sum_{t=1}^{T} P_{S_{0, t} X_{1, t} X_{2, t}}\left(s_{0}, x_{1}, x_{2}\right)-\bar{Q}\left(s_{0}, x_{1}, x_{2}\right)\right|<\epsilon .
$$

Let $Z$ be a random variable that is uniformly distributed over $\{1, \ldots, T\}$ independent of $S_{0}^{T}, S_{1}^{T}, S_{2}^{T}, X_{1}^{T}, X_{2}^{T}, Y_{1}^{T}, Y_{2}^{T}$, and define for each $t$,

$$
\begin{aligned}
& U_{t} \triangleq\left(Y_{2}^{t-1}, S_{2}^{t-1}, S_{2, t+1}^{T}\right) \\
& V_{t} \triangleq S_{1, t+1}^{T} .
\end{aligned}
$$

Let $U \triangleq\left(U_{Z}, Z\right), V \triangleq\left(V_{Z}\right), S_{0} \triangleq S_{0, Z}, S_{1} \triangleq$ $S_{1, Z}, S_{2} \triangleq S_{2, Z}, X_{1} \triangleq X_{1, Z}, X_{2} \triangleq X_{2, Z}, Y_{1} \triangleq$ $Y_{1, Z}, \quad Y_{2} \triangleq Y_{2, Z}$. Denote the probability distribution of $\left(S_{0}, S_{1}, S_{2}, U, V, X_{1}, X_{2}, Y_{1}, Y_{2}\right)$ by $P_{S_{0} S_{1} S_{2} U V X_{1} X_{2} Y_{1} Y_{2}}$, and the probability distribution of $\left(S_{0}, X_{1}, X_{2}\right)$ by $Q_{S_{0} X_{1} X_{2}}$. It can be shown that by these definitions,

$$
P_{S_{0} X_{1} X_{2}}\left(s_{0}, x_{1}, x_{2}\right)=\frac{1}{T} \sum_{t=1}^{T} P_{S_{0, t} X_{1, t} X_{2, t}}\left(s_{0}, x_{1}, x_{2}\right)
$$

and

$$
\begin{gathered}
P_{S_{0} S_{1} S_{2} U V X_{1} X_{2} Y_{1} Y_{2}}\left(s_{0}, s_{1}, s_{2}, u, v, x_{1}, x_{2}, y_{1}, y_{2}\right) \\
\left.=\rho_{0}\left(s_{0}\right)\right\urcorner\left(s_{1}, s_{2} \mid s_{0}\right) P_{U V X_{1} \mid S_{1}}\left(u, v, x_{1} \mid s_{1}\right) \\
\times P_{X_{2} \mid U S_{2}}\left(x_{2} \mid u, s_{2}\right) \Gamma\left(y_{1}, y_{2} \mid s_{0}, x_{1}, x_{2}\right)
\end{gathered}
$$

for some conditional probability laws $P_{U V X_{1} \mid S_{1}}\left(u, v, x_{1} \mid s_{1}\right)$ and $P_{X_{2} \mid U S_{2}}\left(x_{2} \mid u, s_{2}\right)$. In the following, we prove that

$$
I_{P}\left(S_{1} ; U \mid S_{2}\right) \leq I_{P}\left(V ; Y_{2}, S_{2} \mid U\right)-I_{Q}\left(V ; S_{1} \mid U\right) .
$$

Recall (18) and (21) and that $\epsilon>0$ can be chosen arbitrarily small. By continuity, (22) and (23) imply that there must exist a conditional probability distribution $Q_{U V S_{1} S_{2} \mid S_{0} X_{1} X_{2}}$ so that the joint distribution $\bar{Q}_{S_{0} X_{1} X_{2}} \times Q_{U V S_{1} S_{2} \mid S_{0} X_{1} X_{2}} \times \Gamma$ factorizes as

$$
\begin{aligned}
\left.\rho_{0}\left(s_{0}\right)\right\urcorner\left(s_{1}, s_{2} \mid\right. & \left.s_{0}\right) Q_{U V X_{1} \mid S_{1}}\left(u, v, x_{1} \mid s_{1}\right) \\
& \times Q_{X_{2} \mid U S_{2}}\left(x_{2} \mid u, s_{2}\right) \Gamma\left(y_{1}, y_{2} \mid s_{0}, x_{1}\right),
\end{aligned}
$$

and satisfies the mutual information constraint

$$
I_{Q}\left(S_{1} ; U \mid S_{2}\right) \leq I_{Q}\left(V ; Y_{2}, S_{2} \mid U\right)-I_{Q}\left(V ; S_{1} \mid U\right) .
$$

To prove (23), we notice that on one hand,

$$
\begin{aligned}
& \frac{1}{T} \sum_{t=1}^{T} I\left(S_{1, t} ; Y_{2}^{t-1} \mid S_{1, t+1}^{T}, S_{2}^{T}\right) \\
& \quad \stackrel{(a)}{=} \frac{1}{T} \sum_{t=1}^{T} I\left(S_{1, t} ; Y_{2}^{t-1}, S_{1, t+1}^{T} S_{2}^{t-1}, S_{2, t+1}^{T} \mid S_{2, t}\right) \\
& \quad=I\left(S_{1, Z} ; U_{Z}, V_{Z} \mid S_{2, Z}, Z\right) \\
& \quad=I_{P}\left(S_{1} ; U, V \mid S_{2}\right),
\end{aligned}
$$

where $(a)$ follows by the i.i.d-ness of the sequence $\left\{S_{1, t}\right\}$; and the last two equalities by the definitions of $\left(Z, U_{Z}, V_{Z}, S_{1, Z}\right)$ and $\left(U, V, S_{1}\right)$ and the independence of $Z$ and $S_{1}$.
On the other hand, by the definitions of $\left(U_{Z}, V_{Z}, Z, Y_{2, Z}, S_{2, Z}\right)$ and $\left(U, V, Y_{2}, S_{2}\right)$,

$$
\begin{aligned}
\frac{1}{T} \sum_{t=1}^{T} I\left(S_{1, t+1}^{T} ; Y_{2, t} \mid Y_{2}^{t-1}, S_{2}^{T}\right) & =I\left(V_{Z} ; Y_{2, Z} \mid U_{Z}, S_{2, Z}, Z\right) \\
& =I_{P}\left(V ; Y_{2} \mid U\right),
\end{aligned}
$$

By Csiszár's telescoping identity [17], the left-hand sides of (26) and (27) coincide, and thus:

$$
I_{P}\left(S_{1} ; U, V \mid S_{2}\right) \leq I_{P}\left(V ; Y_{2} \mid U, S_{2}\right),
$$

which by chain rule of mutual information and by the Markov chain $(U, V) \multimap S_{1} \multimap-S_{2}$ is equivalent to

$$
\begin{aligned}
I_{P}\left(S_{1} ; U \mid S_{2}\right) & \leq I_{P}\left(V ; Y_{2} \mid U, S_{2}\right)-I_{P}\left(V ; S_{1} \mid U, S_{2}\right) \\
& =I_{P}\left(V ; Y_{2}, S_{2} \mid U\right)-I_{P}\left(V ; S_{1}, S_{2} \mid U\right) \\
& =I_{P}\left(V ; Y_{2}, S_{2} \mid U\right)-I_{P}\left(V ; S_{1} \mid U\right) .
\end{aligned}
$$

\section{REFERENCES}

[1] O. Gossner, P. Hernandez, and A. Neyman, "Optimal use of communication resources," Econometrica, vol. 74, no. 6, pp. 1603-1636, 2006.

[2] B. Larrousse and S. Lasaulce, "Coded power control: performance analysis," in Proc. of ISIT, Istanbul, Turkey, Jul. 2013

[3] P. Cuff and L. Zhao, "Coordination using implicit communication," in Proc. of ITW, Paraty, Brazil, 2011, pp. 467-471.

[4] M. L. Treust, "Empirical coordination for joint source-channel coding," submitted to IEEE Trans. Inf. Theory, 2014. Available: http://arxiv.org/abs/1406.4077.

[5] B. Larrousse, S. Lasaulce, and M. Bloch, "Coordination in distributed networks via coded actions with application to power control," submitted to IEEE Trans. Inf. Theory. Available: http://arxiv.org/pdf/1501.03685.pdf

[6] B. Larrousse, S. Lasaulce, and M. Wigger, "Coordination in state-dependent distributed networks: The two-agent case," submitted to ISIT, Hong Kong, Jan. 2015. Available: http://arxiv.org/pdf/1501.06493.pdf

[7] A. Wyner and J. Ziv, "The rate-distortion function for source coding with side information at the decoder," IEEE Trans. Inf. Theory, vol. 22, no. 1, pp. 1-10, Jan. 1976.

[8] A. H. Kaspi, "Two-way source coding with a fidelity criterion," IEEE Trans. Inf. Theory, vol 31, no. 6, pp. 735?740, 1985.

[9] S. I. Gel'fand "Capacity of one broadcast channel," Probl. Inf. Transmiss., vol 13, no. 3, pp. 106-108, 1977.

[10] H. Asnani, H. Permuter, and T. Weissman, "Successive refinement with decoder cooperation and its channel coding duals," accepted to IEEE Trans. Inf. Theory. Available: http://arxiv.org/abs/1203.4865

[11] Y. Cemal and Y. Steinberg, "The multiple-access channel with partial state information at the encoders," IEEE Trans. Inf. Theory, vol. 51, no. 11, pp. 3392-4003, Nov. 2005.

[12] Y. Steinberg and S. Shamai (Shitz), "Achievable rates for the broadcast channel with states known at the transmitter," in Proc. ISIT, Adelaide, Australia, Sept. 4-9, 2005, pp. 2184-2188.

[13] O. Shayevitz and M. Wigger, "An achievable region for the discrete memoryless broadcast channel with feedback," Proc. ISIT 2010, Austin, Texas, June 13-18, 2010.

[14] R. Gray and A. Wyner, "Source coding for a simple network," Bell System Tech. J. vol. 48, pp. 1681-1721, Nov. 1974

[15] H. Yamamoto, "Source coding theory for cascade and branching communication networks", IEEE Trans. Inf. Theory, vol. 27, no. 3, pp. 299-308, May 1981.

[16] P. H. Cuff, H. H. Permuter, T. Cover, "Coordination capacity," IEEE Trans. Inf. Theory, vol. 56, no. 9, pp. 4181-4206, Sep. 2010.

[17] A. El Gamal and Y.-H. Kim, "Network information theory," Cambridge University Press, 2011. 